\section{Asbestos-the Silent Killer: a review of screening and diagnostic imaging of asbestos related lung disease}

\author{
Ali Nawaz Khan, ${ }^{1}$ Hamdan Al-Jahdali, ${ }^{2}$ \\ Klaus L. Irion, ${ }^{3}$ Shyam Sunder Koteyar ${ }^{1}$ \\ 'North Manchester General Hospital, \\ Pennine Acute NHS Trust, Manchester, \\ UK; ${ }^{2}$ King Saud University for Health \\ Science, King Adulaziz Medical City, \\ Riyadh, Saudi Arabia; ${ }^{3}$ The Cardiothoracic \\ Centre Liverpool NHS Trust, The Royal \\ Liverpool University Hospital, UK
}

\section{Abstract}

An epidemic of asbestos related lung (ARLD) disease stares the developed countries in the face. However, these countries have lately banned the use of asbestos. Nevertheless, Western countries have no qualms of selling asbestos to poor countries that have no credible health safety regulations in the work place. Epidemiologic studies predicted a decline in incidence of ARLD in the US after the year 2000 , with a peak incidence in the UK in 2020. Lung cancer (LC) develops in up to $25 \%$ of asbestos workers. In exposed nonsmokers, there is 5 -fold increase. In exposed individuals, smoking further increases the risk of bronchogenic carcinoma by $80-90$-fold. However the silent killer, asbestos, is still active in developing countries and is likely to remains so in decades to come. In the Middle East, immigrant workers still work in asbestos environment. Saudi Arabia is the only major country in the Middle East that has banned asbestos, but workers continue to be at risk due to maintenance work on buildings built before the ban. As asbestos related lung disease remains silent in many and has a long latent period, the only way of detecting these diseases early is by diligence being aware of the patient's occupational history and affective imaging. Presently there is no credible screening for ARLD. Here we review imaging studies in ARLD to increase awareness of this potentially lethal disease. Whereas diagnosis of ARLD is a matter for compensation in the developed countries it is a matter of survival in the developing world where industrialization has just begun and may take decades to remove the threat of asbestos. In this review we will discuss the clinical, radiologic, and pathologic features of ARLD.

\section{Introduction}

Asbestos has been widely used as a building material and other uses for decades. This hazardous substance is currently banned in countries such as Australia, Argentina, Chile, Croatia, and Saudi Arabia. Several countries, such as Brazil, Russia and Canada do still mine asbestos, and in the United States, the substance is strictly regulated, but it is not totally banned. Between 1940 and 1979, asbestos was used almost exclusively for its fire resistant properties. Unfortunately, asbestos can still be found in roofing, siding, walls, ceilings, floors, pipes, boilers, gaskets, cloth and blankets, protective clothing, cement, firebrick, gunnite, and hot tops. Its sounds ludicrous, but between 1952 and 1956, R Lorillard Company produced 12 billion Kent cigarettes with asbestos filters. Series of advertisements, placed in the JAMA \& the New England Journal of Medicine, stressed the health protection Kent's gave. The filtering material was a top wartime secret, which represented the greatest scientific advance ever made in cleansing air of impurities. ${ }^{1}$ High exposures ceased in the US in the late 1970s,
Correspondence: Hamdan Al-Jahdali, King Saud University for Health Science, King Adulaziz Medical City, Riyadh, Saudi Arabia.

E-mail: jahdali@yahoo.com

Key words: asbestosis, malignant pleural mesothelioma, pleural plaques, asbestos related lung cancer, imaging asbestos related lung disease.

Received for publication: 29 June 2011. Accepted for publication: 8 September 2011.

This work is licensed under a Creative Commons Attribution NonCommercial 3.0 License (CC BYNC 3.0).

\section{CCopyright A.N. Khan et al., 2011}

Licensee PAGEPress, Italy

Chest Disease Reports 2011; 1:e13

doi:10.4081/cdr.2011.e13

and later in the UK, because of legislation passed. However, because the latency period between an initial exposure and the development of ARLD is 20 years or longer, asbestos related lung (ARLD) disease remains an important public health issue..$^{2,3}$

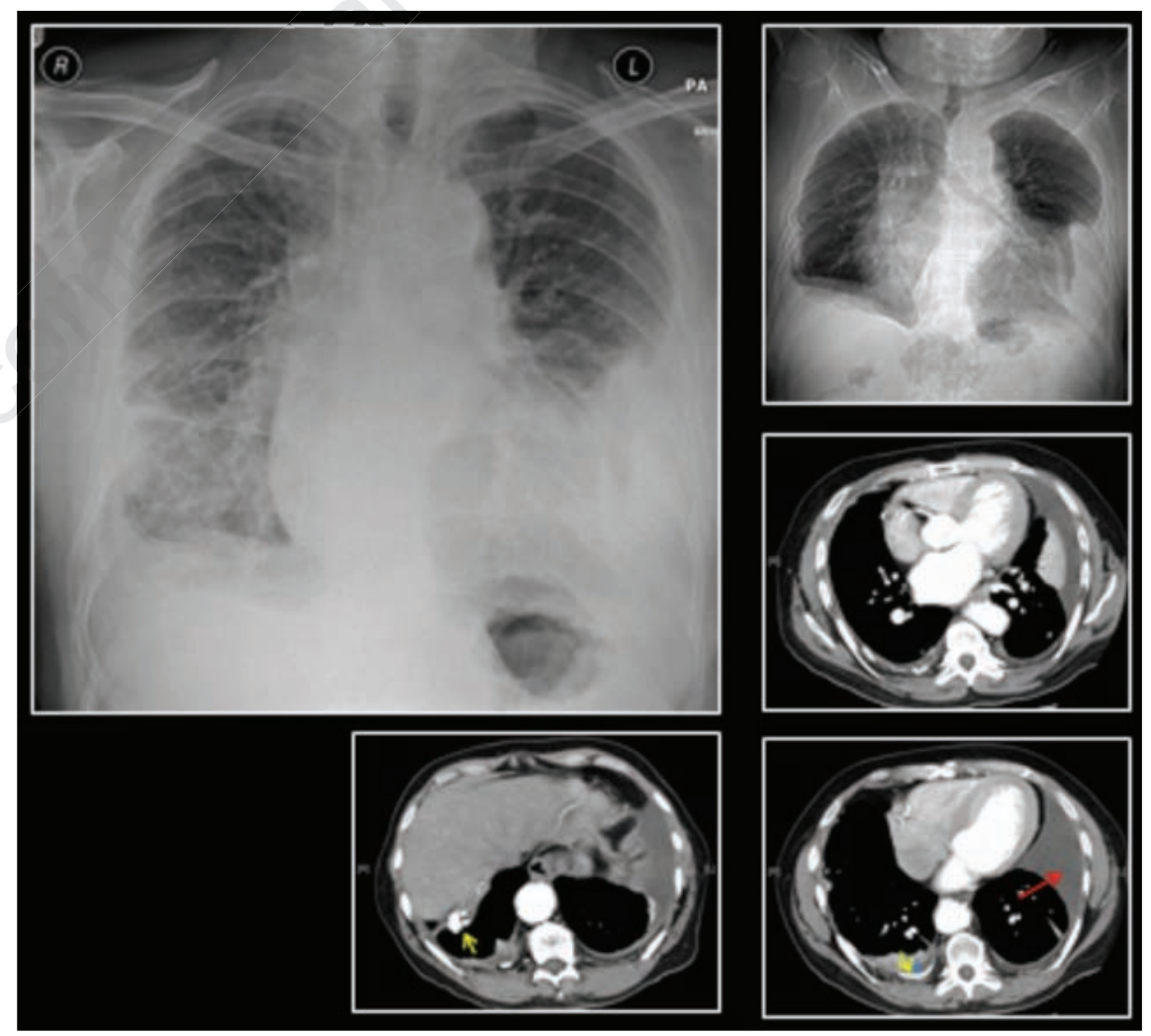

Figure 1. Asbestos related diffuse pleural thickening could mimic pleural effusion and even heart failure as on this radiograph. The scanogram top left confirms the CXR findings. An ultrasound examination of the thorax failed to show a pleural effusion. A guided pleural biopsy revealed extensive pleural fibrosis. Note the diffuse pleural fibrosis resembles a loculated effusion (red arrow). The associated multiple pleural plaques (yellow arrows) are not readily apparent on the radiograph. 


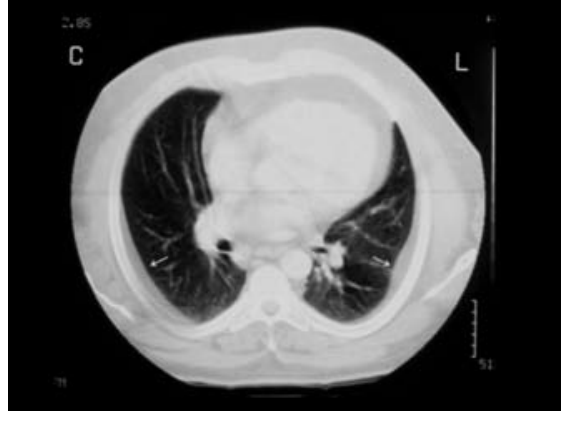

Figure 2. Extrapleural fat as in this case may mimic diffuse pleural thickening.
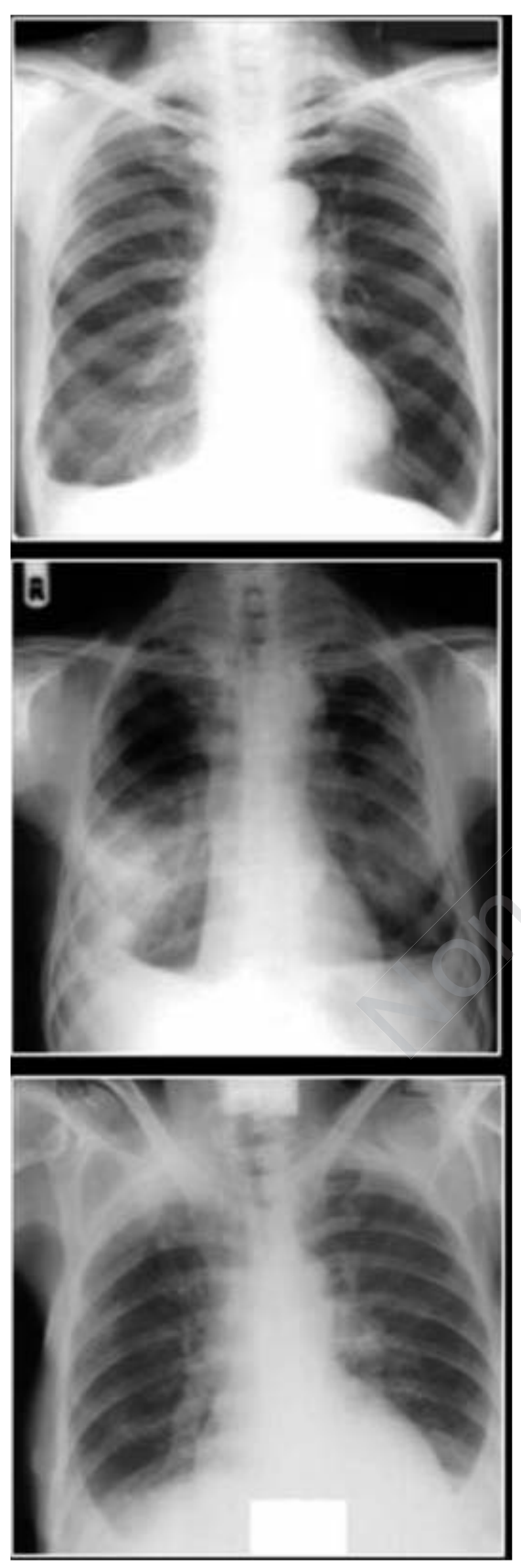

Figure 3. Asbestos related benign pleural effusion may unilateral or bilateral as seen in these three separate patients
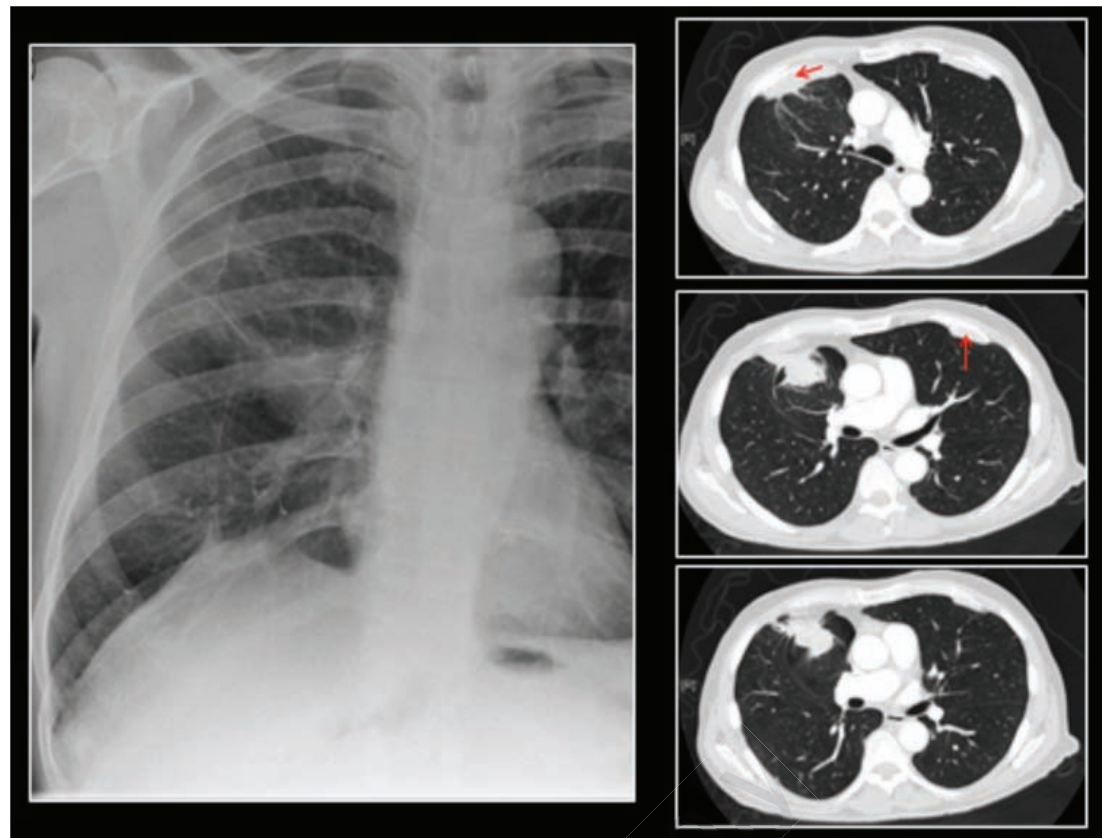

Figure 4. The diagnostic features of round atelectasis include contiguity to areas of diffuse pleural thickening, a lentiform or wedge-shaped outline, evidence of volume loss in the adjacent lung, and a characteristic comet tail of vessels and bronchi sweeping into the margins of the mass. Additional CT features include crowding of air bronchograms and the presence of a hurricane sign, which is a curvilinear bronchovascular bundle leading into the mass. Note calcified pleural plaques (red arrows). Note also the close relationship to the lesser fissure.

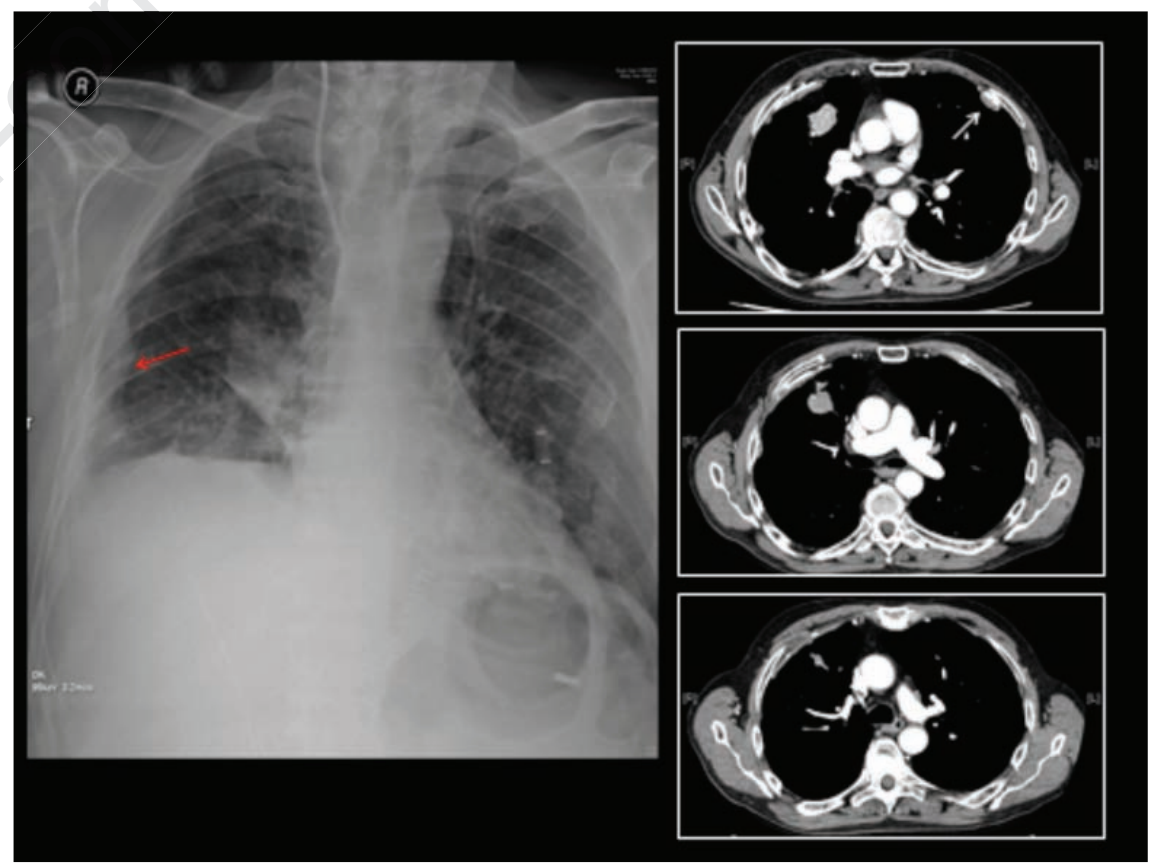

Figure 5. Asbestos related round atelectasis: note the lentiform opacity in close relation with the lesser fissure associated with both calcified pleural plaques (White arrows) and diffuse pleural thickening (red arrow) and evidence, of volume loss in the right lung.

[Chest Disease Reports 2011; 1:e13] 


\section{Discussion}

The diagnostic approach to ARLD is different from that of other diffuse lung diseases because of the medico-legal implications. The likelihood of ARLD should be determined, and other causes eliminated. An assessment of the extent of disease is used to calculate compensation. ${ }^{4}$

Malignant mesothelioma (MPM) rarely occurs in patients with no history of exposure to asbestos. ${ }^{5-10}$ Other asbestos related pathology include Lung cancer (LC), benign pleural effusion (BPE), discrete pleural plaques or diffuse benign pleural fibrosis (DPP) and rounded atelectasis (RA). The imaging hallmark of asbestos exposure is the presence of the calcified pleural plaques (CPP) where, bilateral diaphragmatic calcification with costophrenic angle sparing is considered pathognomonic. MPM is an important differential diagnosis for BPE although the two are not linked. Diagnosis of a BPE is based on history of asbestos exposure, exclusion of other causes of pleural effusion, and the absence of malignancy for 3 years after the onset of the effusion. Although both BPE and MPM are related to asbestos exposure, BPE tends to occur earlier than MPM, with an onset approximately 10 years after exposure versus approximately 20 40 years 11 .

MPM arises from mesothelial cells of the pleural and peritoneal cavity including pericardium and tunica vaginalis testis. Over $90 \%$ patients with MPM are men. Asbestos exposure is an established cause. The WHO classifies mesothelioma into three histological types: epithelioid, sarcomatoid and biphasic malignant mesothelioma. The histological type is an important prognostic marker. The longest survival is seen in patients with epithelioid MPM. The worst outcome has been described with sarcomatoid subtype. MPM share many pathological similarities with benign pleural lesions and other malignancies. Additional immunohistochemistry is essential in histopathological assessment using a marker panel of antibodies. ${ }^{12}$ Low pleural fluid glucose a high $\mathrm{C}$ - reactive protein and the presence of pleural fluid and pleural thickening are poor prognostic indicators with MPM. ${ }^{13}$ Diagnosis and prognosis of MPM is highly dependent on accurate histological interpretation of pleural biopsies. Smaller biopsies can cause difficulties in characterizing pleural lesions, and immunostains are required. ${ }^{14}$ Endoscopic ultrasound-guided fine needle aspiration (EUS-FNA) can be used in the diagnosis of a MPM to determine negative N2 stage lymph nodes as these patients can benefit from extra pleural pneumonectomy with adjuvant therapy. A positive N2 lymph node by EUS-FNA may be a contraindication to definitive surgery in
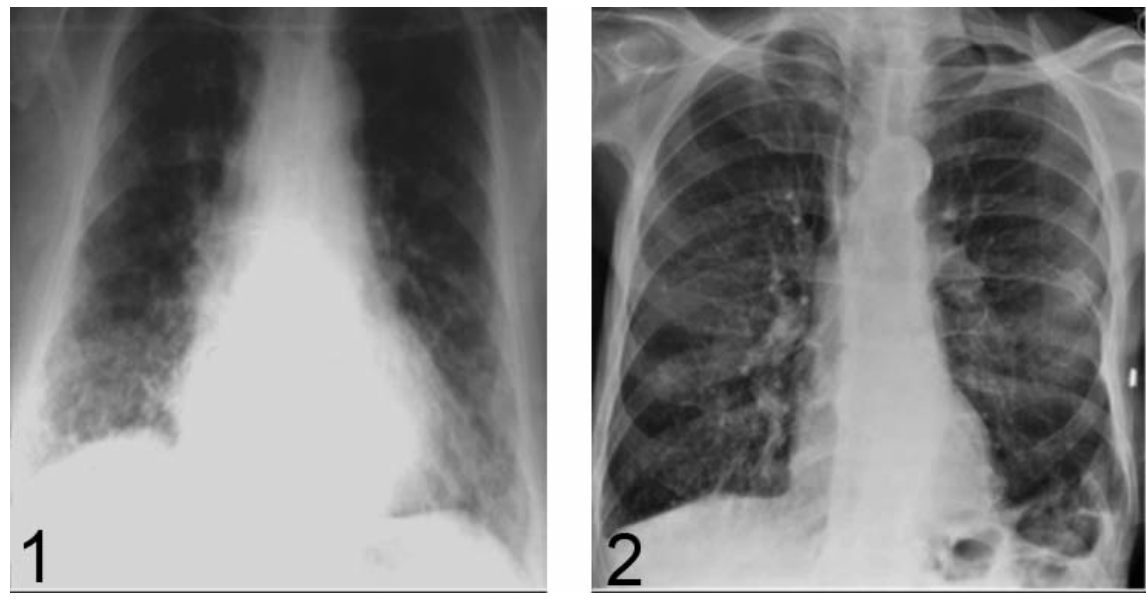

Figure 6. Asbestoses in two separate patients. CXR 1 shows reticulonodular shadowing predominantly at the lung bases. CXR 2 show more extensive reticular shadowing associated with shaggy, hemidiaphragms and multiple pleural plaques.

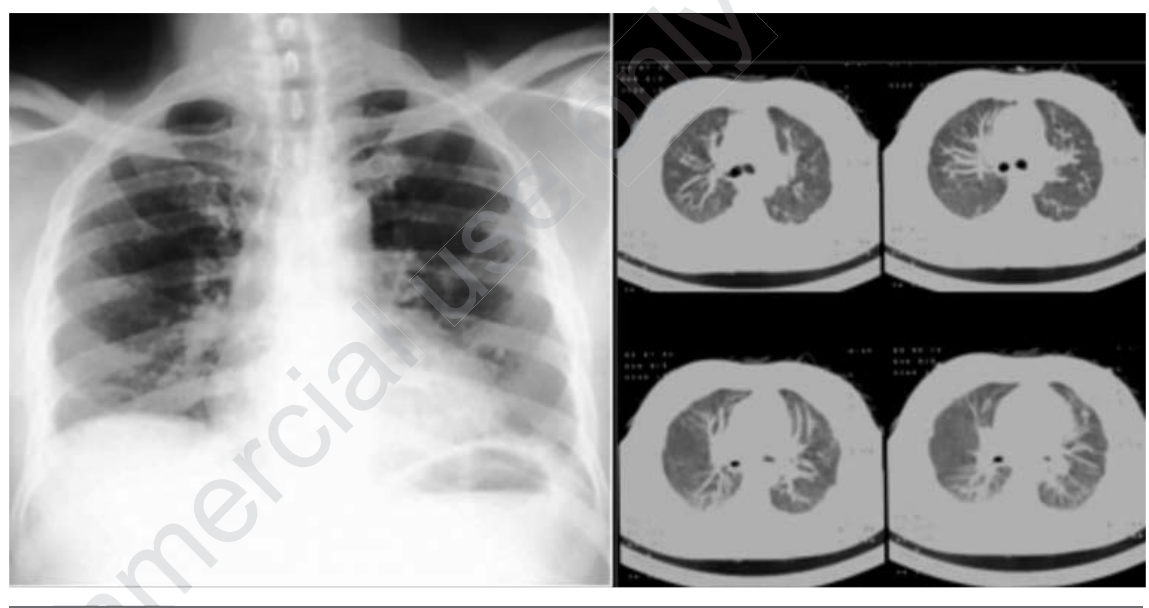

Figure 7. Ground-glass attenuation on HRCT is a relatively unusual feature of asbestosis. When present, it may represent edema or fine intralobular fibrosis. Note that CXR may pass for normal.

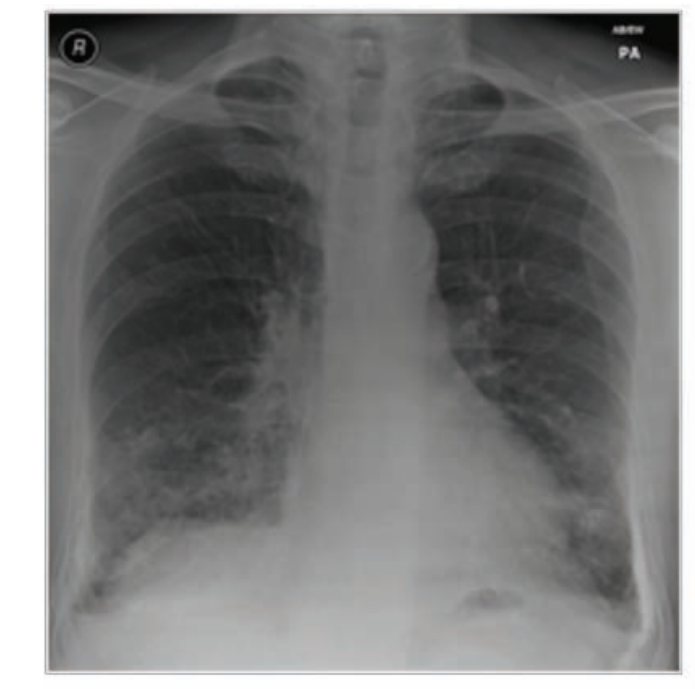

Figure 8. HRCT in asbestoses show subtle interlobular nodular septal thickening (white arrow), parenchymal bands (red arrow) and not calcified and non-calcified pleural plaques (green arrows).
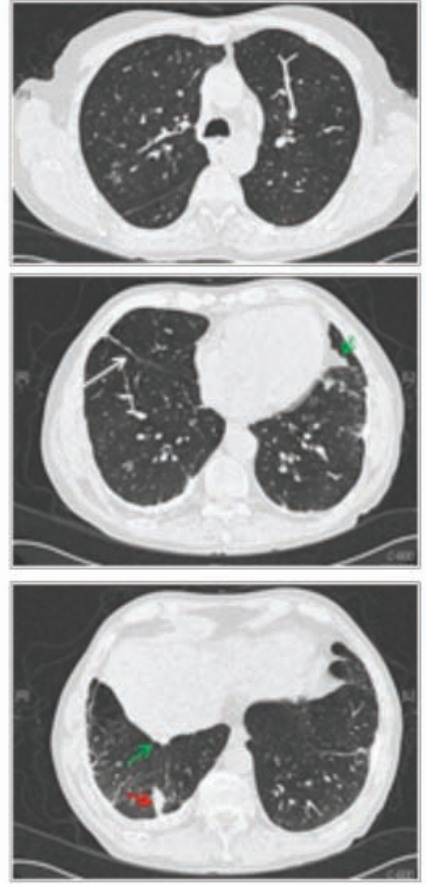
patients with MPM. ${ }^{15}$ Histological subtype determination is subject to sampling error, but an adequate specimen obtained from surgical biopsy increases the accuracy compared with radiological-guided biopsies. ${ }^{16}$ Thoracentesis is the first step in a newly discovered pleural effusion. Certain tests such as a natriuretic peptide assay significantly improves the accuracy of a diagnosis of cardiac pleural effusion, whereas PF mesothelin levels greater than 20 nmol/L are highly suggestive of MPM. ${ }^{17}$ Thoracoscopy is a cost-effective and reliable technique for obtaining histological diagnosis, exclude pulmonary embolism and allows a direct pleurodesis when indicated. ${ }^{18}$ Tissue sampling from suspected malignant pleural disease produce a far higher yield when a biopsy is image guided than a blind pleural biopsy. Cutting needles appear to have a higher diagnostic yield in a mesothelioma compared with fine needle aspiration. Image guided biopsies have a lower complication rate. Biopsy tract seeding appears lower using smaller biopsy ports, as used for image-guided pleural biopsy. ${ }^{19}$ Asbestos-related diffuse pleural thickening (DPT), due to extensive fibrosis of the visceral pleura is common in asbestos workers. DPT may be associated with dyspnoea and chest pain. It causes a restrictive defect on lung function and may rarely result in respiratory failure and death. DPT may coexist with asbestos related pleural plaques but has a distinctly different pathology. BPE commonly antedate the development of DPT. 11,20

\section{Screening for asbestos related lung disease}

The methods for diagnosing ARLD are invasive and unsuitable for an increasingly elderly population. New non-invasive methods such as analysis of exhaled breath biomarkers e.g. exhaled nitric oxide (ENO), exhaled breath condensate or of exhaled volatile organic compounds could potentially be extremely useful. ${ }^{21}$ The benefit of radiographic screening/CT for ARLD remains uncertain. Presently a CXR remains the modality of choice but diagnosis is difficult in early stages. Moreover, there is a significant inter-observer variation in interpreting CXR in asbestos workers despite improvements in the International Labour Office (ILO) classification system..$^{22}$ It has been suggested that high resolution CT (HRCT) be added a part of international classification. ${ }^{22}$

\section{Radiography}

Asbestosis is interstitial pulmonary fibrosis secondary to the presence of intrapulmonary

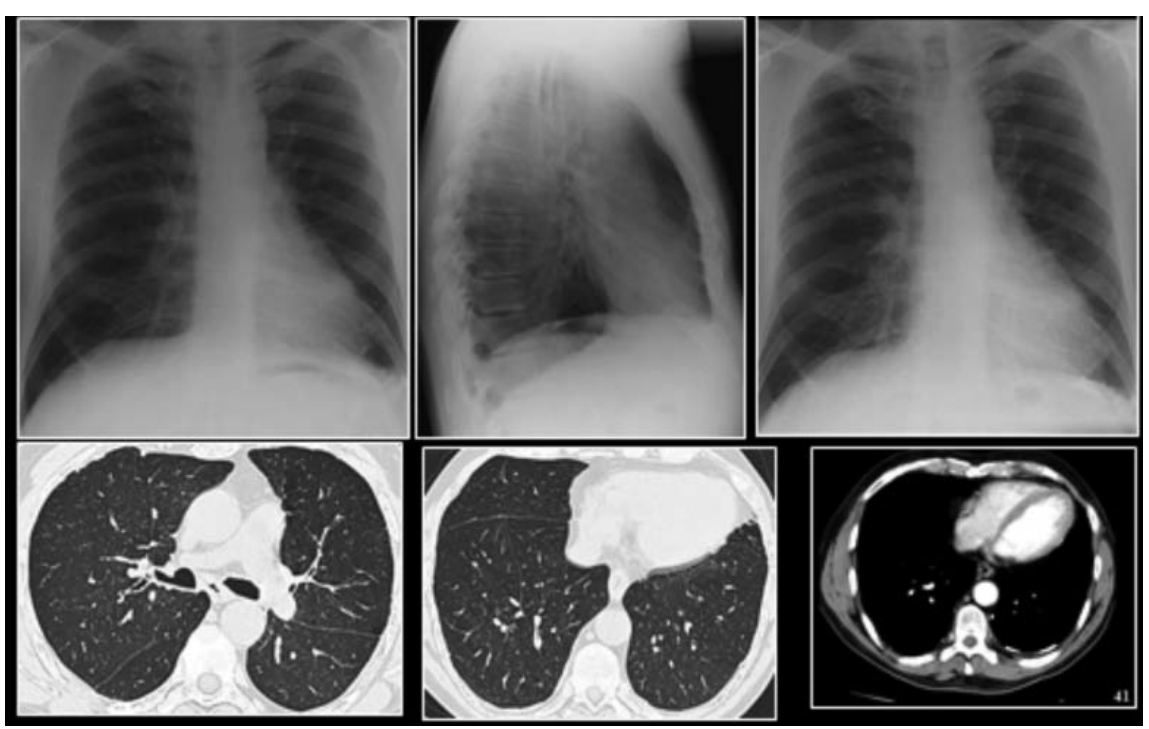

Figure 9. HRCT in asbestos related lung disease shows subtle ground glass appearance and multiple pleural plaques.

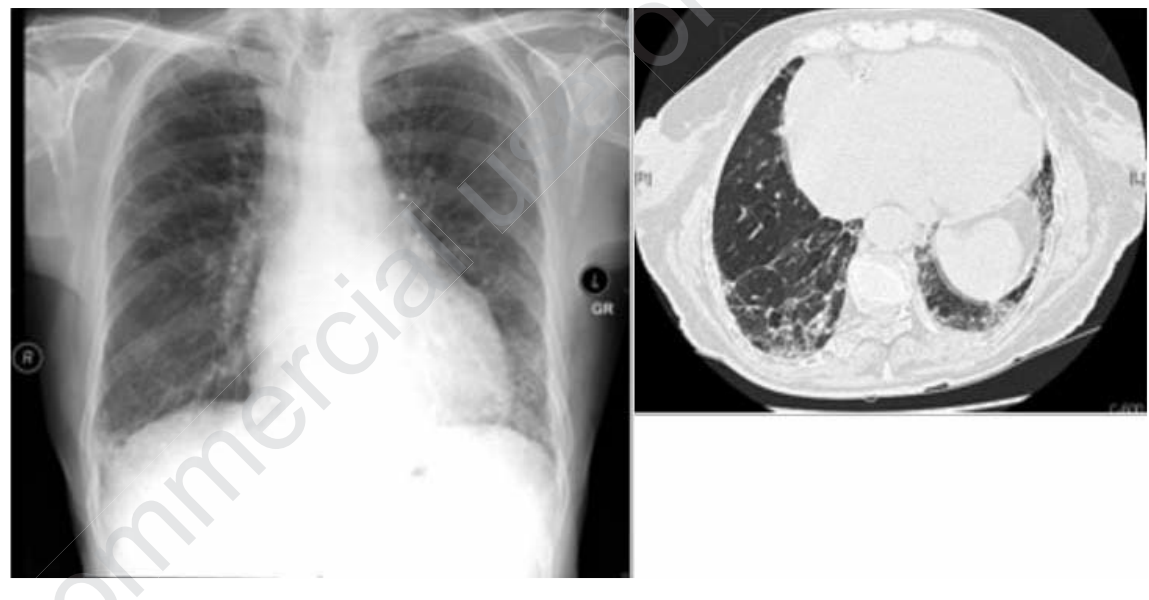

Figure 10. High-resolution CT scan shows subpleural pleural fibrosis amounting to honeycombing in asbestoses. Note the similar appearance on the CXR.

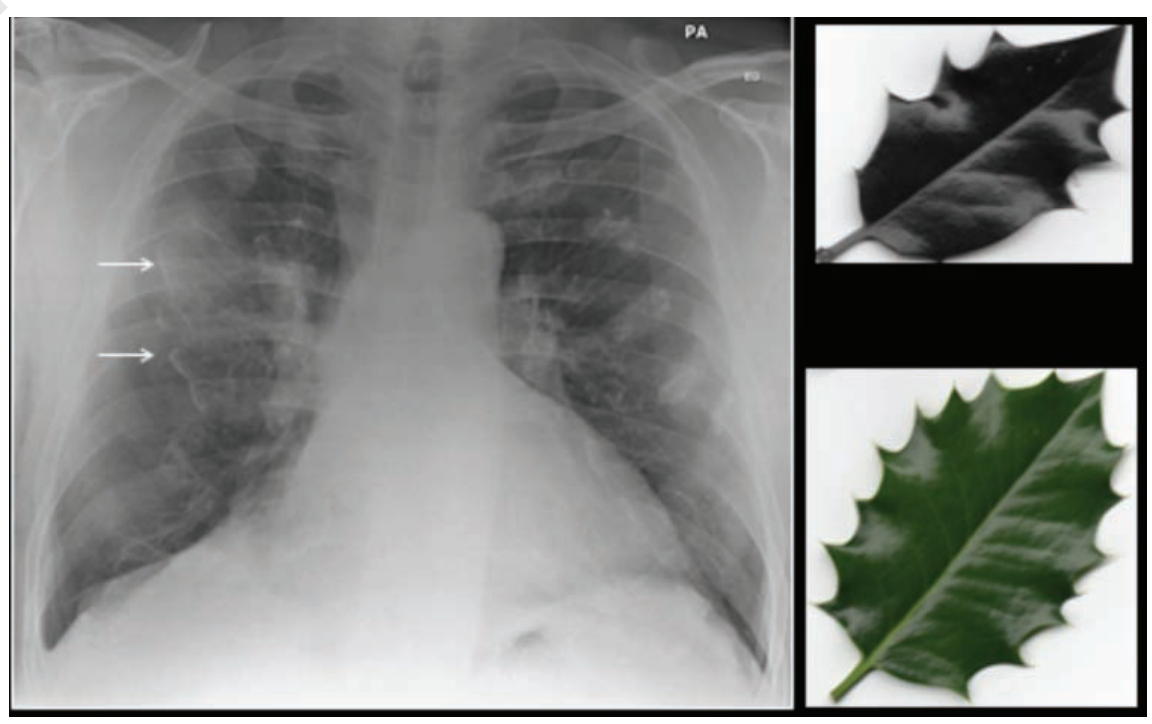

Figure 11. Asbestos related calcified pleural plaques showing the holly leaf appearances. Holly leaves are shown on the right. 
asbestos bodies or asbestos fibers. The findings on a CXR include ground-glass opacification, small nodules, shaggy cardiac borders, and ill-defined diaphragmatic contours. The fibrosis is usually most severe in the subpleural lower zones. CXR findings include fine reticular opacities and septal lines that progress towards a coarser linear pattern of honeycombing with advanced disease. Ancillary findings of curvilinear subpleural lines and parenchymal bands, which are linear opacities $2-5 \mathrm{~cm}$ long extending from or paralleling the pleural surface, may be seen. These are characteristic, although not specific. The lines and bands represent contiguous, thickened interlobular septa, which are areas of subsegmental atelectasis or fibrosis along bronchovascular bundles. Mild mediastinal lymphadenopathy, believed to represent a form of reactive hyperplasia occurs frequently in patients with uncomplicated asbestosis (Figures 1-5).

\section{Discrete pleural plaques}

Discrete pleural plaques (DPP) may be seen in profile or 'en plaque' and are classically seen on a CXR along the postero-lateral thoracic wall, the dome of the diaphragm, and the mediastinal pleura. In profile, plaques appear as focal, smooth opacities, usually less than 1 $\mathrm{cm}$ thick, paralleling the chest wall. Appearances en plaque is of a poorly defined opacity with irregular margins. Plaques may be isolated and usually spare the visceral pleura, lung apices, and costophrenic angles. In profile, calcified plaques appear as opaque lines parallel to the chest wall, mediastinum, pericardium, and diaphragm. En face, calcified plaques are irregular, heterogeneous densities, the so-called holly leaf. The presence of bilateral, superior diaphragmatic surface calcifications with clear costophrenic angles is virtually pathognomonic for asbestos-related pleural disease and considered diagnostic of asbestos exposure (Figures 6-12). ${ }^{23,24}$

\section{Diffuse pleural thickening}

Asbestos related diffuse pleural thickening (DPT) is smooth, uninterrupted pleural opacity extending over at least one quarter of the chest wall, with or without obliteration of the costophrenic angles. DPT is at least $5 \mathrm{~cm}$ wide, 8-10 cm long cranio-caudally, and $3 \mathrm{~mm}$ thick. Proliferation of extrapleural fat is a frequent finding with DPT. Unlike discrete pleural plaques, DPT may be associated with significant reduction in pulmonary function. The diagnosis of DPT may be difficult on a CXR and differentiation between DPT and focal pleural plaques may be problematic. However, diffuse pleural thickening due to asbestos exposure rarely calcifies, tends to involve the costophrenic angles (unlike plaques), tends to be ill defined, and is more extensive than focal
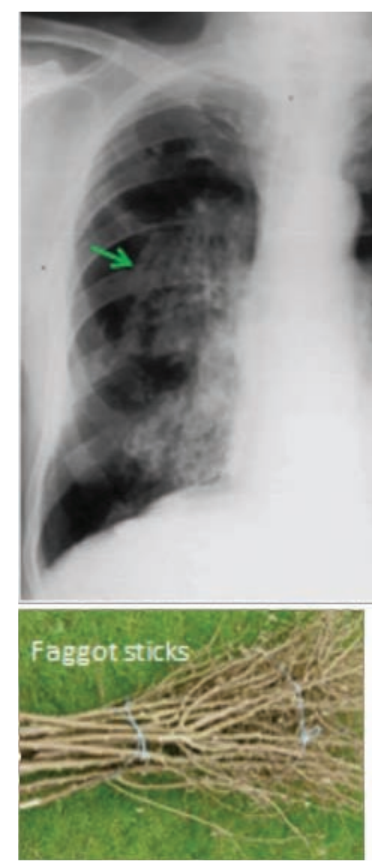
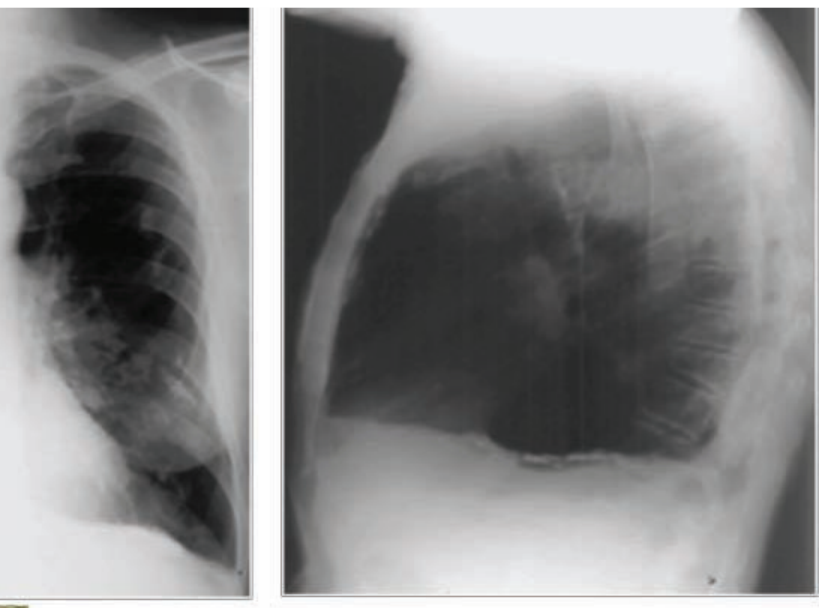

Figure 12. Benign asbestos pleural calcification resembling a bunch of faggot sticks (green arrow).
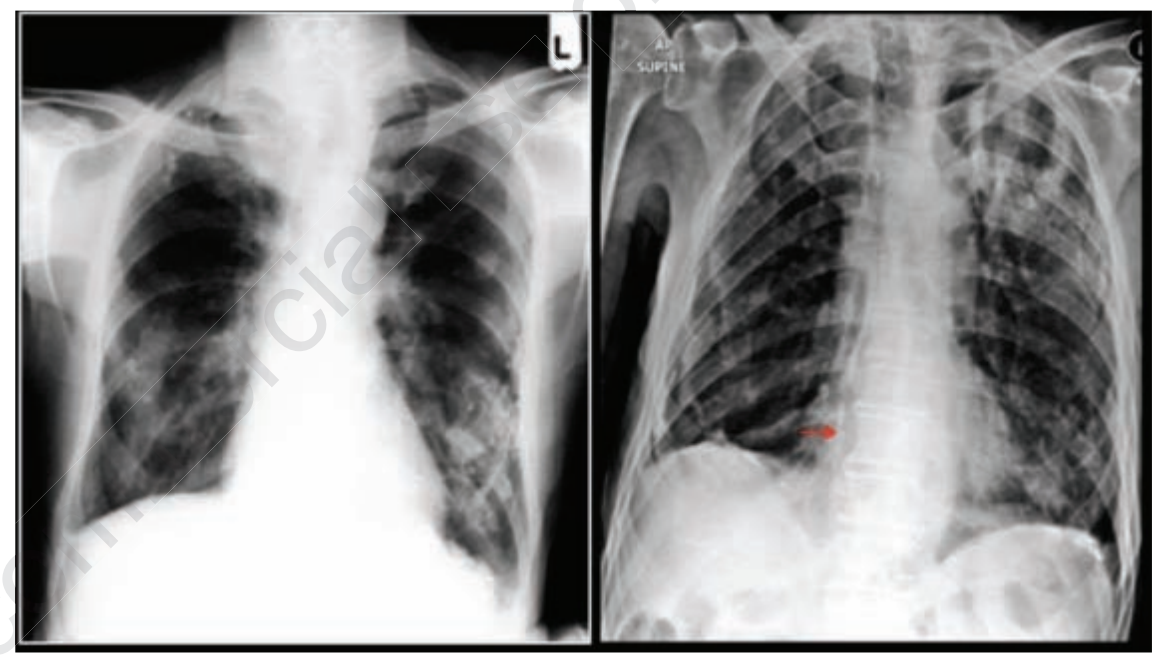

Figure 13. Asbestos related calcified pleural plaques can appear in various shapes and sizes, some linear others amorphous but they tend to have sharp corners and almost geometrical outlines. The CXR on the left besides other calcified pleural plaques show calcification of the posterior spinal pleural reflection (red arrow).

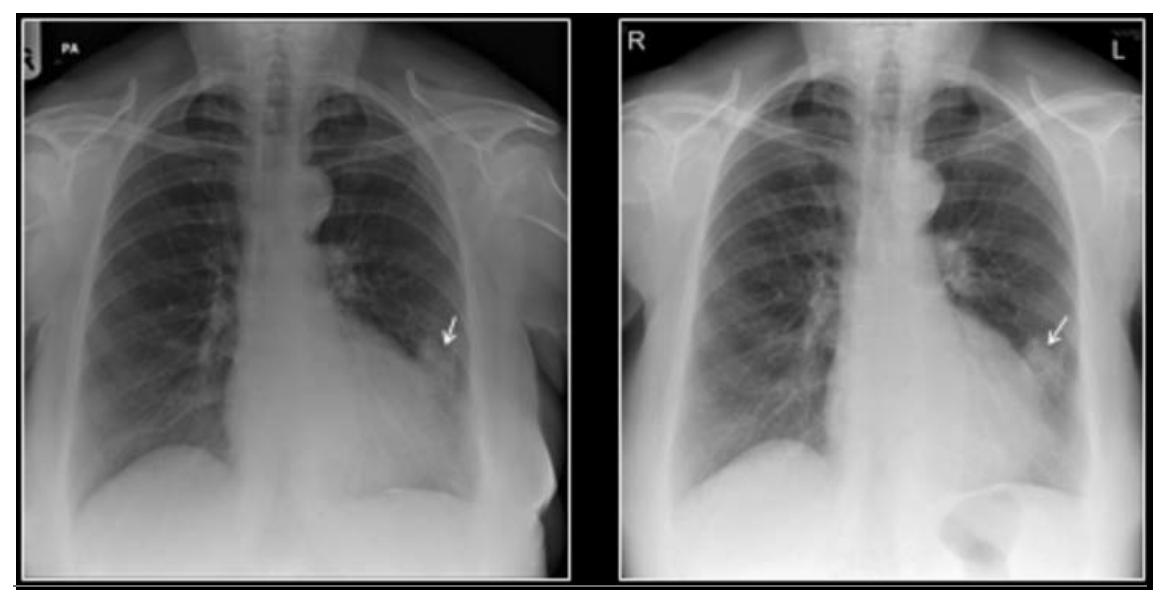

Figure 14. Solitary asbestos related plaques might remain stable over years; the two radiographs are 7 years apart that show a single plaque in the left paracardiac region. 
plaques. Involvement of interlobar fissures is common. DPT is associated more often with radiologically detectable asbestosis than are pleural plaques (Figures 13-15).

\section{Benign pleural effusion}

Benign pleural effusion (BPE) is a diagnosis of exclusion. BPEs are usually small, they may be unilateral or bilateral, and they tend to resolve spontaneously over 3-4 months, although they may recur. BPE, greater than 500 $\mathrm{mL}$ are uncommon. Half of BPE subsequently develop pleural thickening and may antedate RA. CT scanning is useful to help exclude an underlying cause or associated pleural mass. ${ }^{25}$ Some effusions are associated with pleural plaques (Figure 16).

\section{Rounded atelectasis}

Rounded atelectasis (RA) appears as a welldefined, rounded, focal, sub pleural soft-tissue mass of 2-7 cm in diameter that abuts an area of pleural thickening. Most atelectasis is located in the posterolateral or posteromedial parts of the lower lobes. Bilateral lesions occur. Mild volume loss may be associated. The appearances usually remain stable over time, but occasionally, masses may increase or decrease in size. The most important differential diagnosis of RA is bronchogenic carcinoma. Biopsy may be necessary. RA is not specific for asbestos exposure and may be preceded by pulmonary infarction, Dressler syndrome, heart failure, and nonspecific pleural effusions (Figures 17,18). ${ }^{26}$

\section{Lung cancer}

Asbestos related lung cancer is more prevalent at the lung periphery and lung bases but overall the appearances are no different from cancers not associated with asbestos exposure. The most common radiographic finding is a pulmonary mass with associated mediastinal lymphadenopathy (Figures 19,20). ${ }^{27}$

\section{Malignant mesothelioma}

Malignant mesothelioma (MPM) are generally irregular, nodular, diffuse pleural thickening, occasionally associated with a pleural effusion. Less commonly, MPM presents as an isolated effusion or pleural mass. As MPM progresses it may encase the entire hemithorax, encasing the whole lung. Mediastinal shift to the effected side occasionally occurs. Other features of ARLD such as pleural plaques are seen in only approximately $20-25 \%$ of patients. Rarely a MPM may present with a spontaneous pneumothora (Figures 21,22,23,24,25,26). ${ }^{28}$

Conventional radiography has many limitations in the diagnosis of ARLD. The quality of the CXR and the size, shape, position, and degree of calcification determines the detection of pleural plaques. While the detection of

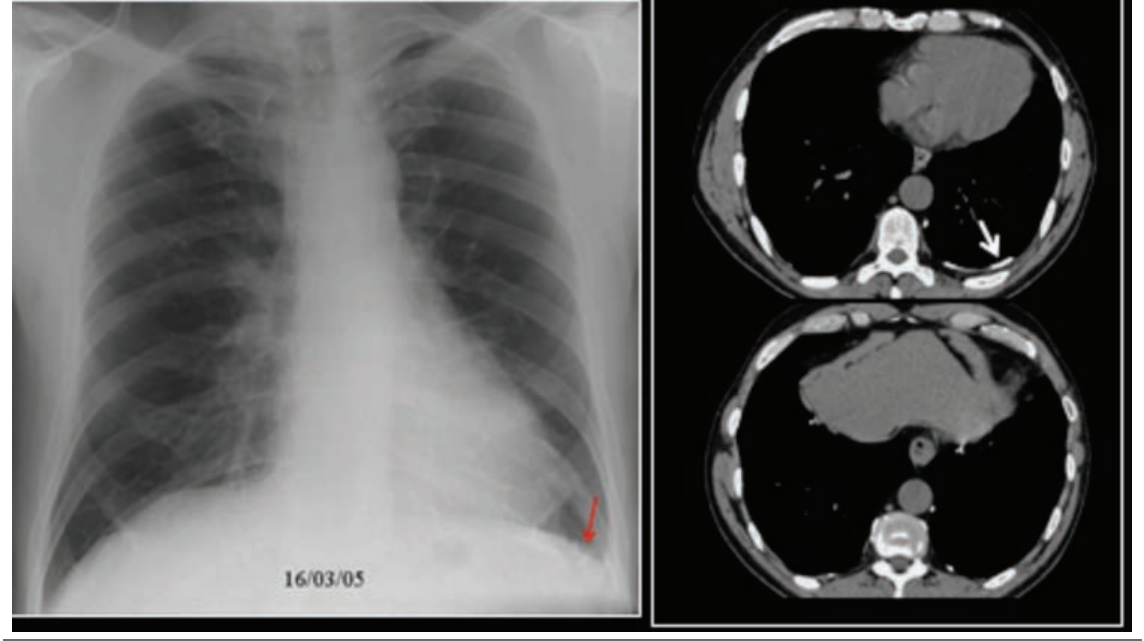

Figure 15. Benign calcified pleural plaques: Although on the CXR, the superior surface of the left hemidiaphragm looks shaggy (red arrow) but no definite calcified plaques are identified. The unenhanced CT scans performed within a weak following the CXR show definite bilateral calcified pleural plaques (white arrow).

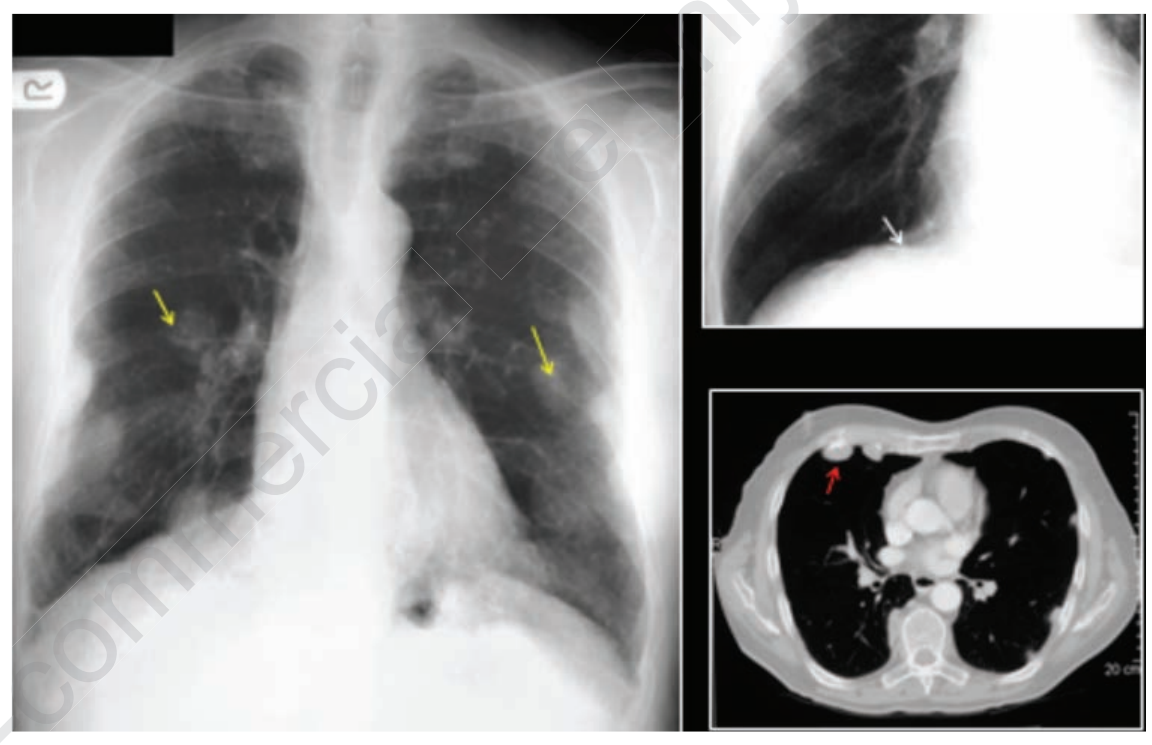

Figure 16. Extensive pleural plaques mimicking lung metastases in this patient staged for intra-abdominal malignancy (yellow arrows). A magnified view of the left hemidiaphragm (top right) depicts supradiaphragmatic calcified pleural plaques. An axial CT scan reveals the true nature of the multiple masses seen on the CXR as benign pleural plaques (red arrows).

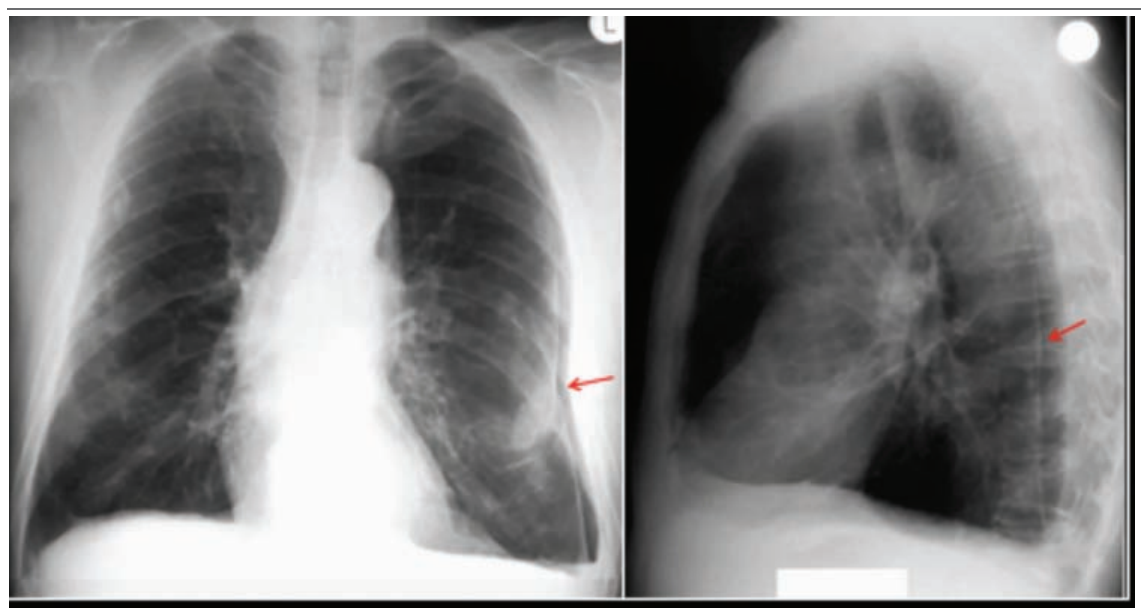

Figure 17. Asbestos related pleural calcification presenting as a pneumothorax. Note the shaggy hemidiaphragms. 


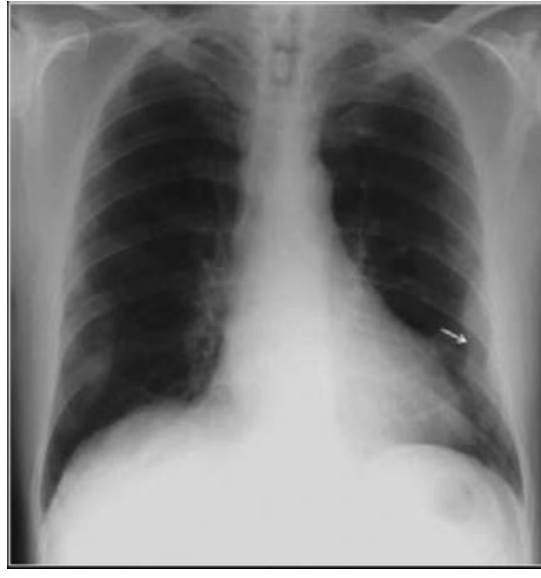

Figure 18. Asbestos related diffuse pleural thickening shown along both lower chest walls, note involvement of the left costophrenic angle.
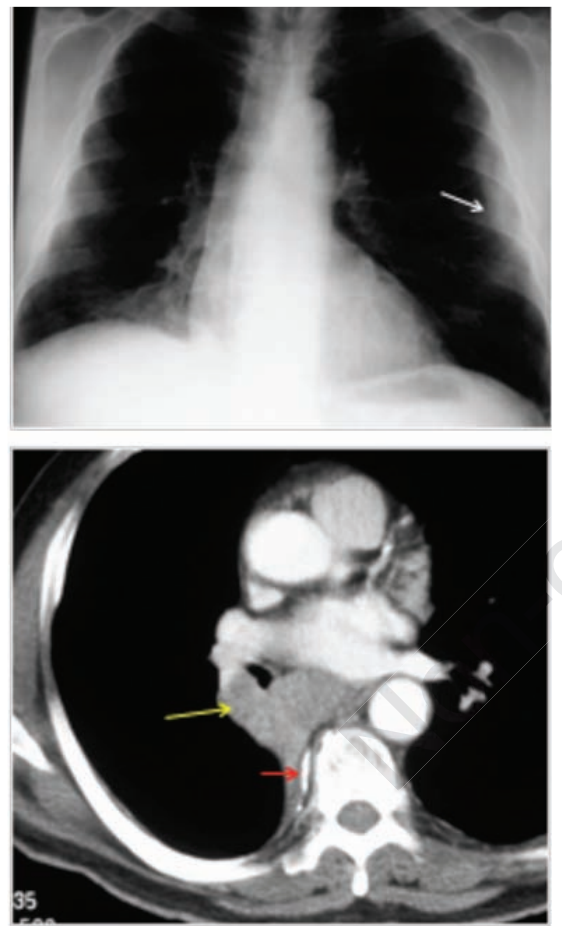

Figure 19. Asbestos related lung cancer in a 60-year-old that was a smoker and had worked with textiles for 40 years. The CXR show vague shadowing at the left cardiophrenic angle not indicative of a neoplasm. Note the diffuse pleural thickening (arrow). However, since the patient presented with a small history of hemoptysis a CT examination was carried out. The CT shows right hilar mas associated with posterior mediastinal lymphadenopathy (yellow arrow) note the calcified pleural plaque. The patient expired 4 months later. A post mortem examination revealed a squamous bronchial carcinoma with features of asbestos exposure.

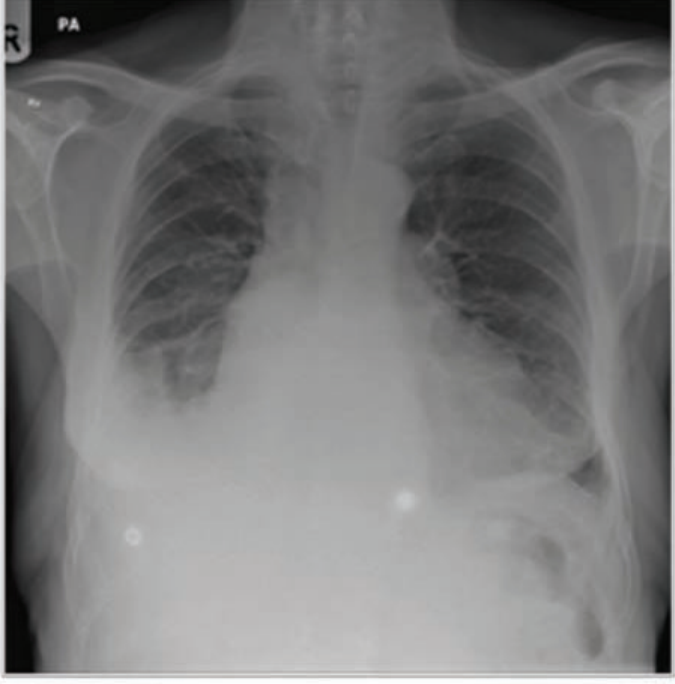

Figure 20. Asbestos related lung cancer in a patient with asbestoses (biopsy proven), calcified pleural plaques, noncalcified pleural plaques, small pericardial/pleural thickening (red arrow) histological proven adenocarcinoma (yellow arrow) and right basal diffuse pleural thickening and an incidental hiatus hernia $(\mathbf{H})$.
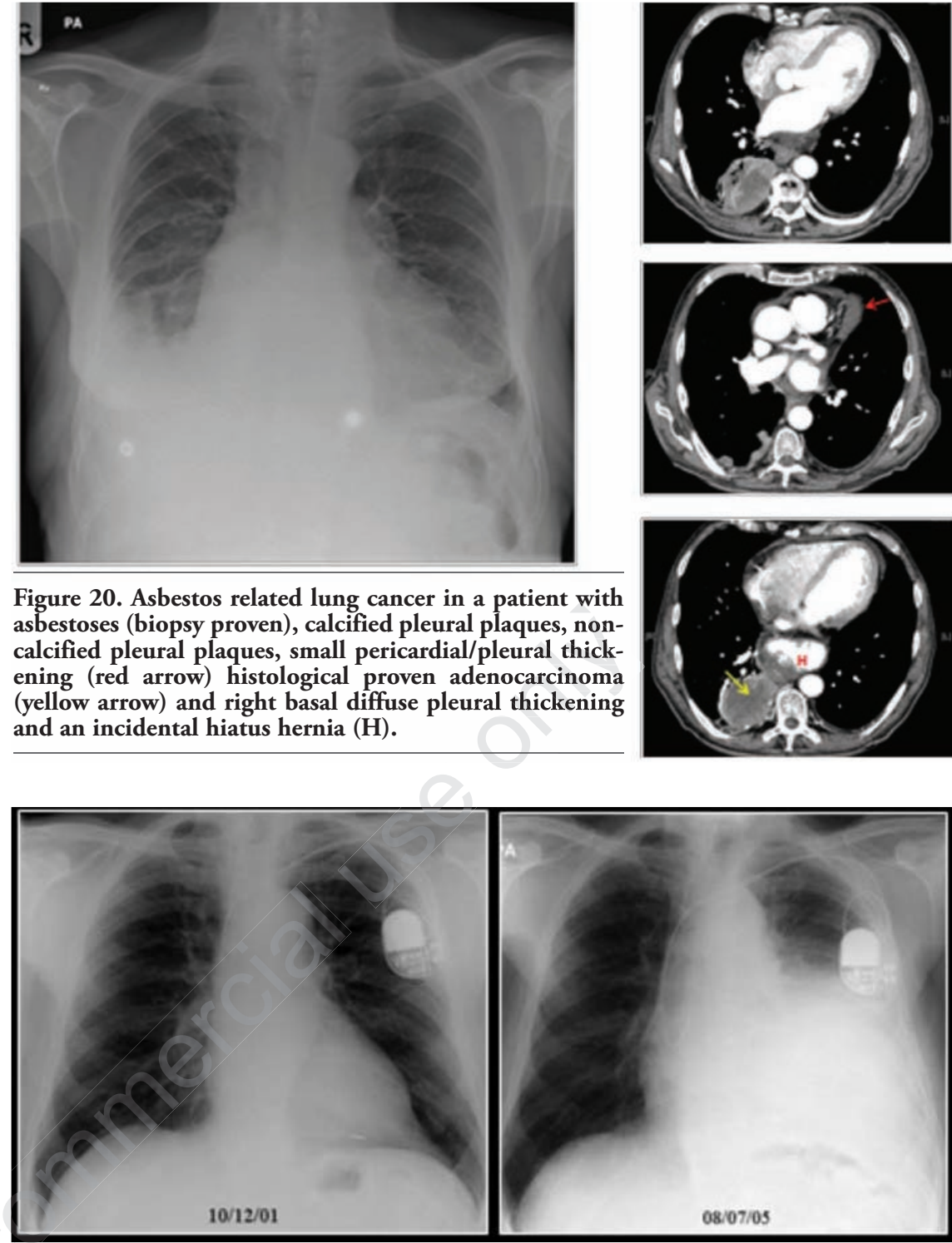

Figure 21. Conventional CXRs 4 years a part show features of a left sided pleural effusion. The appearances appeared suspicious of heart failure. However, because of previous asbestos exposure an ultrasound scan and subsequently a CT scan was organised (see Figure 22).

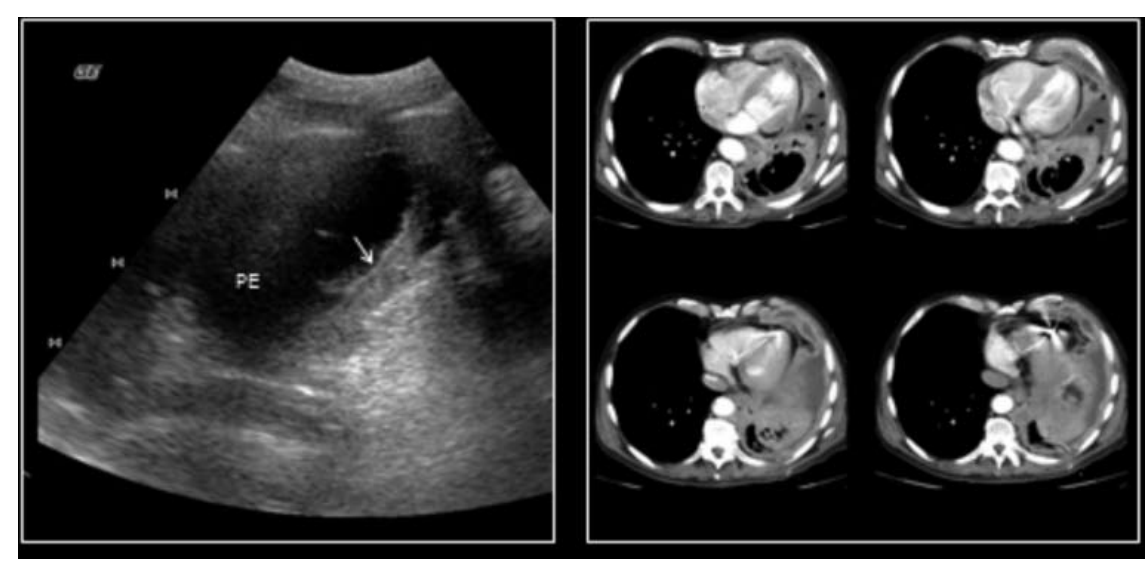

Figure 22. Images on the same patient as in Figure 21 shows pleural thickening (white arrow) associated with a pleural effusion on the ultrasound examination (PE). The CT scans confirm these findings. An ultrasound guided biopsy revealed a malignant pleural mesothelioma. 
multiple, bilateral, randomly scattered, calcified, costal, and diaphragmatic pleural plaques is virtually diagnostic of asbestos exposure, there is $11 \%$ false-positive rate with CXR. Extrapleural fat mimics pleural thickening (Figure 15) and is a significant cause of falsepositive outcome. A high false negative rate has also been reported. CT is more specific and more sensitive than a CXR in the diagnosis of asbestos-related pleural disease. .9,30 $^{29}$ Furthermore, as many as $20 \%$ of patients with histologically proven ARLD have normal CXR findings, and $80 \%$ of patients with radiographic findings of mild disease have histologic results of moderate or severe fibrosis. ${ }^{31}$ A CXR often underestimates the extent of MPM and is associated with significant false positive and false negative rates. There is a high inter-observer variability rates in the detection of pleural plaques. Prominent subpleural fat or normal rib companion shadows may mimic focal or diffuse pleural thickening, leading to false-positive diagnoses in as many as $20 \%$ of patients. Plaques may be difficult to differentiate from DPT. However, plaques usually spare the costophrenic angles and apices and rarely extend over more than the fourth rib interspace, while DPT rarely calcifies and is usually more irregular and ill defined.

\section{Computer Tomography}

Computer Tomography (CT) scanning is an established criterion standard in the evaluation of pleural disease. High resolution CT (HRCT) is more specific and sensitive than a CXR in the assessment of asbestosis particularly on images taken in the prone position. ${ }^{32-37}$ However, HRCT scan findings are not specific for asbestosis. Thus, it has a questioned specificity. HRCT features of asbestoses include bilateral pulmonary fibrosis and bilateral pleural plaques or diffuse pleural thickening in an individual with an appropriate history of exposure (Figures 2-5). HRCT is also valuable in excluding disease in individuals with equivocal CXR findings. CT is of value in differentiating benign disease from malignant pleural disease. The presence of a contiguous sheet or pleural rind, pleural nodularity, and thickening greater than $1 \mathrm{~cm}$, as well as the involvement of mediastinal pleura, are findings suggestive of malignancy. However, case reports also report a variant of asbestos-related, diffuse pleural thickening that appears nodular and that is radiologically indistinguishable from mesothelioma. In equivocal cases, biopsy is needed. HRCT diagnosis of pleural plaques is not without pitfalls. Normal extrapleural fat internal to the ribs, particularly postero-laterally from the fourth-to-eighth ribs extending into the costophrenic angles may mimic pleu-

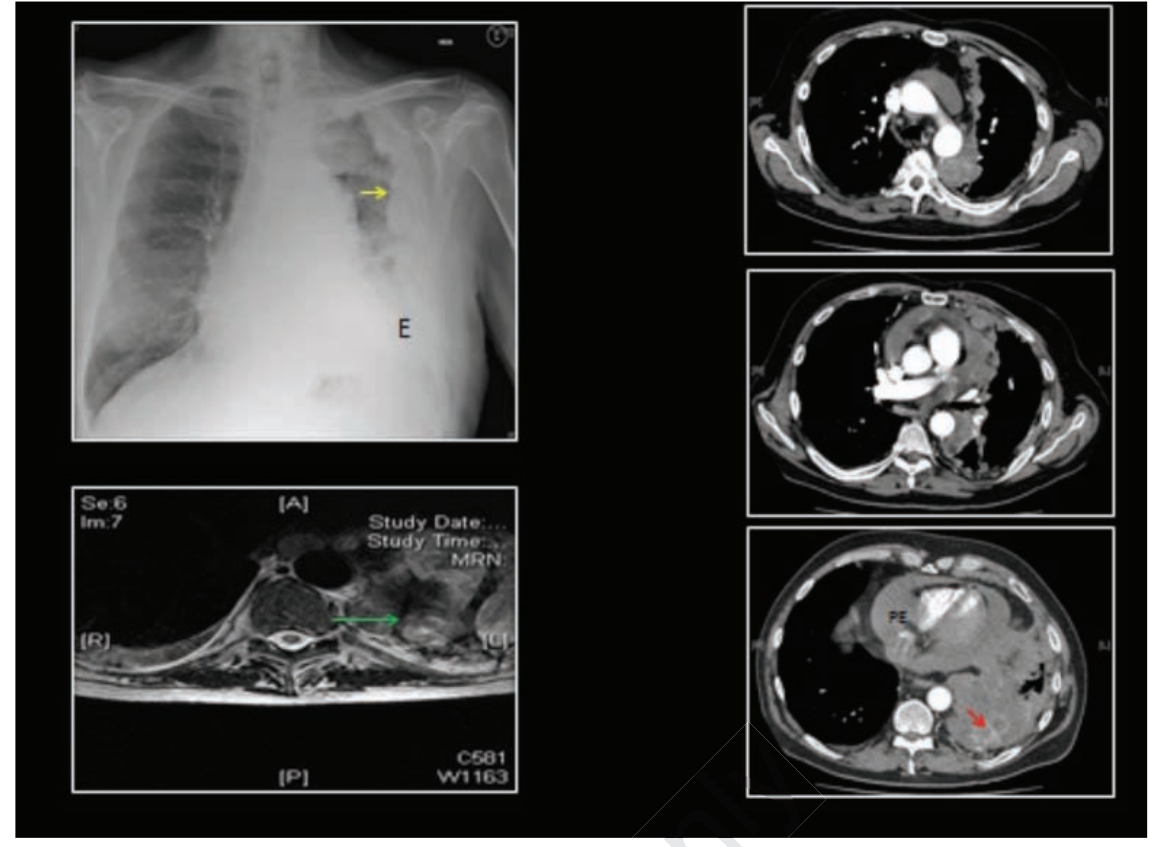

Figure 23. A PA chest radiograph shows left-sided lobulated thickening (yellow arrow) and pleural effusion (E), in a patient exposed to asbestos. These findings are regarded as characteristic of malignant mesothelioma. The axial contrast enhanced CT scans shows an enhancing pleural mass (red arrow), meditational invasion associated with a pericardial effusion (PE). The axial T2 MRI at the level of the lung bases show a complex signal pleural mass suggestive of a pleural tumor (green arrow). The pleural biopsy showed patchy papillary hyperplasia of the surface mesothelium. Underlying stroma was focally infiltrated by malignant cells forming glands and trabeculae. Immunostains for calretinin and CK5/6 were strongly positive within the cells. Ber-EP4 and TTF-1 are negative. The PSA, S100, CK7, CK20 and Cdx-2 stains were negative. The histological appearances were suggestive of a malignant mesothelioma. There was no evidence of lymphovascular permeation.

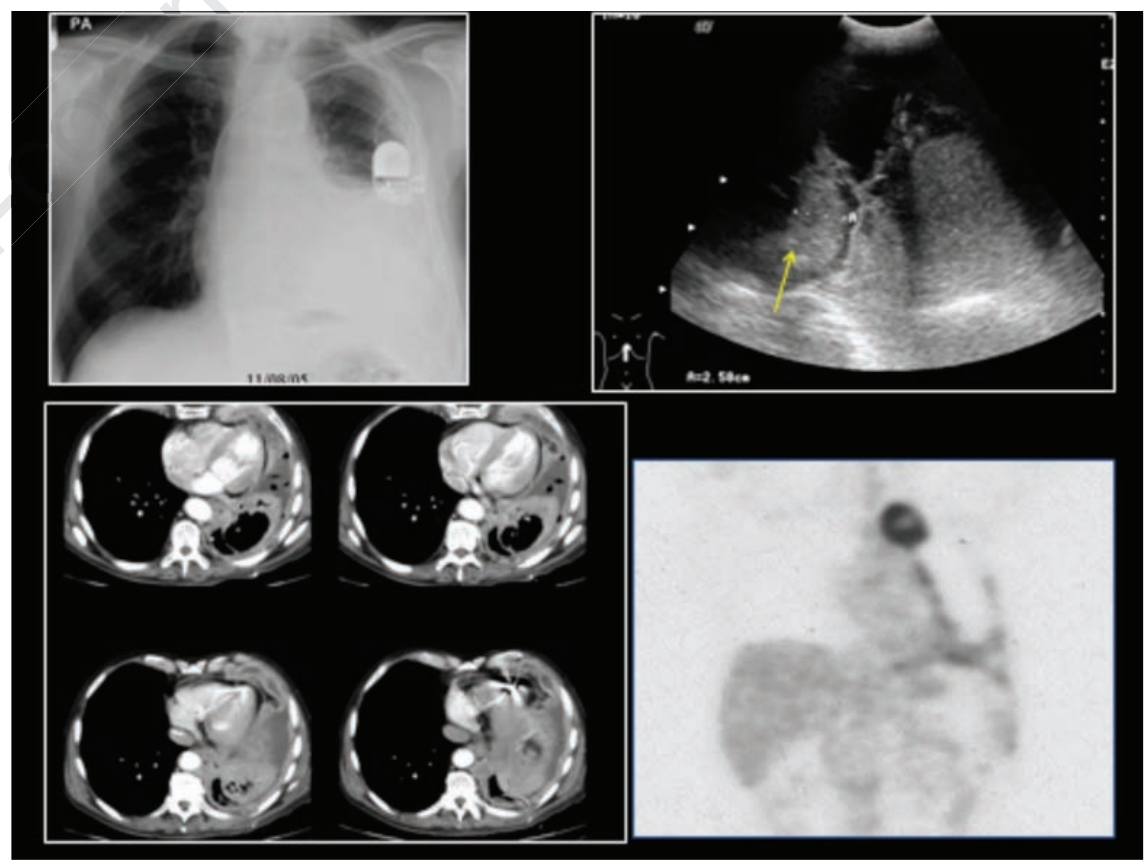

Figure 24. Images show the value of different modalities in the diagnosis of malignant pleural mesothelioma. The ultrasound scan shows a solid pleural mass surrounded by pleural fluid. An FDG-PET scan shows increased isotope uptake at the left lung base, which appear to be encasing the left lower hemithorax. Note the loss of lung volume on the coronal FDG PET scan. 
ral plaques when extended windows are used. The pitfall can be avoided by the use of soft tissue windows. Fat can be several millimeters thick. A number of anatomic structures that may mimic pleural plaques are the normal transversus thoracis and subcostalis muscles, segments of intercostal veins, visceral pleural thickening, and confluent subpleural nodules. The transversus thoracis and subcostalis muscles usually are smooth, of uniform thickness, and bilaterally symmetrical. ${ }^{38}$

Intercostal vessels may cause spurious appearances of focal pleural thickening (Figure 15). Intercostal vessels can occasionally be traced to the azygos or hemi-azygos veins an observation that allows the correct interpretation. Moreover, extrapleural fat should be visible between the vessel and the pleura. When the images are read on the lung window setting, intercostal segments do not indent adjacent lung, while pleural plaques invariably indent. Furthermore, pleural plaques usually are visible over several contiguous intercostal segments and may contain calcification. ${ }^{38}$

Asbestoses are indistinguishable from idiopathic pulmonary fibrosis at a CXR, HRCT, and pathologic examination; the only distinguishing feature is the presence of asbestos bodies.

The diagnostic features of RA include contiguity to areas of diffuse pleural thickening, a lentiform or wedge-shaped outline, evidence of volume loss in the adjacent lung, and a characteristic comet tail of vessels and bronchi sweeping into the margins of the mass. Additional CT features include crowding of air bronchograms and the presence of a hurricane sign, which is a curvilinear bronchovascular bundle leading into the mass. ${ }^{39,40}$ Close surveillance and careful interpretation of CT and HRCT can obviate more invasive procedures (Figures 17,18).

CT is the mainstay in the evaluation of MPM; however, it often underestimates early chest wall invasion, peritoneal involvement, and nodal metastasis (Figures 10,22,23,27). Perfusion CT is an innovative technique that can evaluate the microvasculature of tumors however; it is limited by high radiation exposures and potential toxicity from iodinated contrast media. CT findings in MPM include irregular, nodular pleural thickening, which may involve the interlobar fissures, pleural effusion, and loss of volume, pleural calcification, and chest wall invasion. Pleural thickening is typically nodular and is usually greater than $1 \mathrm{~cm}$ thick, circumferentially involving the parietal and visceral costal and mediastinal pleura. Rarely, MPM may present as a localized masse. MPM often spreads to involve the underlying lung, causing thickening of interlobular septa and parenchymal nodules. MPM may invade the thoracic wall, mediastinum or spread through the diaphragm. Mediastinal lymphadenopathy occurs, although distant
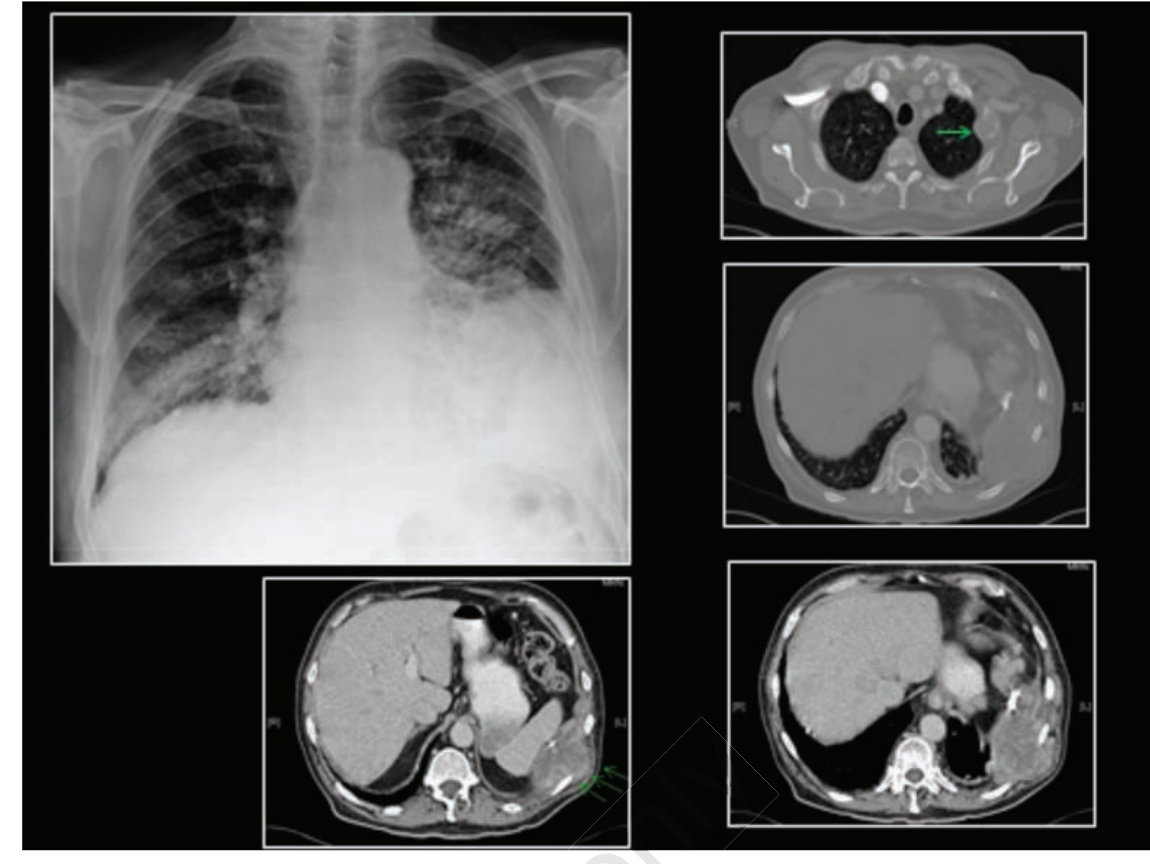

Figure 25. This 75-year-old man with known exposure to asbestos presented with a left lower thoracic pain. Examination revealed a boggy intercostal mass on the lower left postero-lateral aspect of the lower thorax. The CXR shows a left lower thoracic mass/effusion. The axial CT scans show a left lower thoracic mass invading the intercostal space (three arrows) at higher level there is destruction of a rib associated with pleural/extrapleural mass. Note the calcified pleural plaques. An image guided pleural biopsy revealed a malignant pleural mesothelioma. This patient also had vertebral metastases as shown in Figure 26.

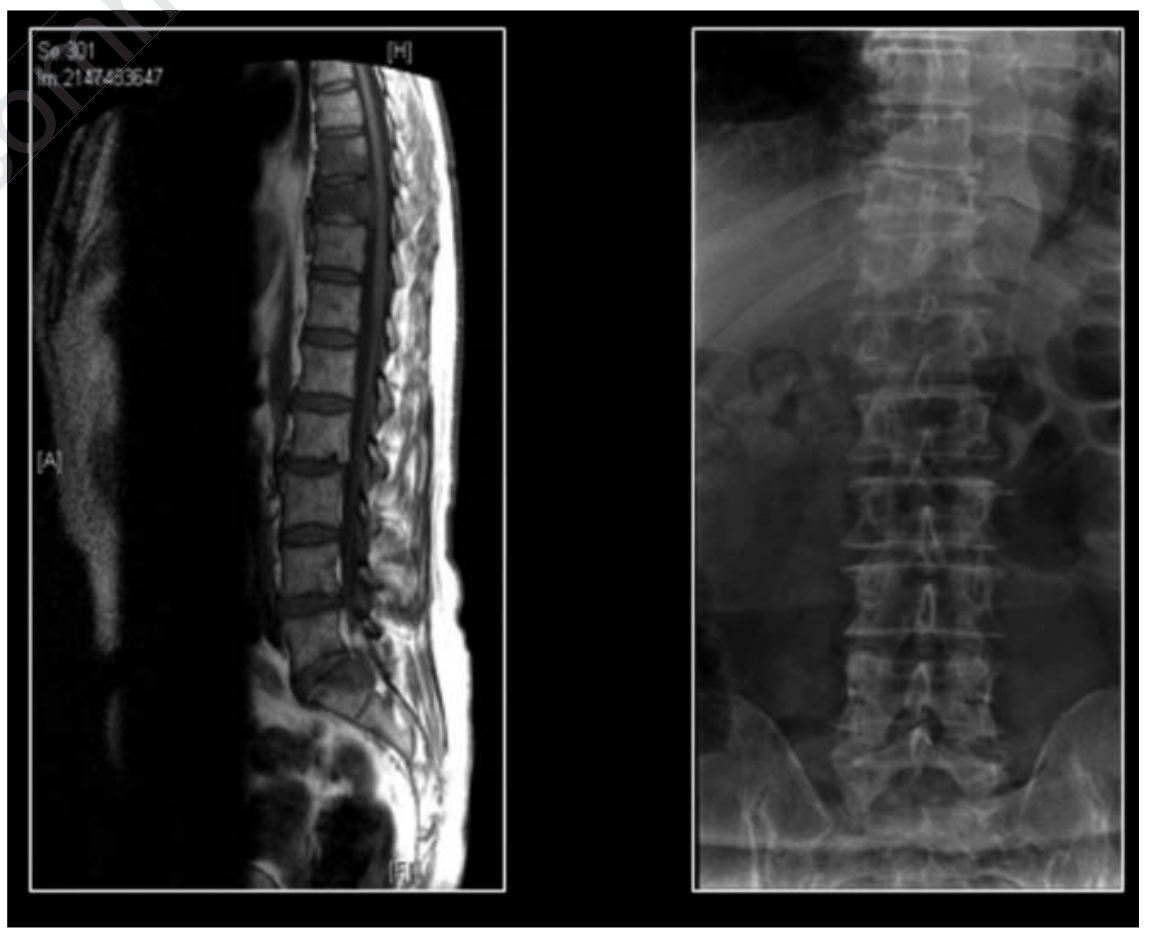

Figure 26. Plain AP lumbosacral spine $x$-ray and corresponding $T 1$ weighted sagittal MR scans show features of bony metastases of D9 and D12. The MRI shows an additional deposit at S1. These images are the patient seen in Figure 25. 
metastases, including hematogenous spread to the contralateral lung, are uncommon. ${ }^{41}$

Compared with A CXR, CT is more sensitive and specific for the detection of diffuse pleural thickening. ${ }^{29-30}$ Furthermore, interobserver agreement in assessing pleural disease is greater with CT scanning than with a CXR.

\section{Magnetic resonance imaging}

MRI is helpful for distinguishing progressive massive fibrosis from lung cancer ${ }^{42}$ and has a complementary role in the evaluation of pleural effusions and MPM. MRI is more sensitive than HRCT in showing pericardial thickening in ARLD. ${ }^{43}$ MRI and CT scanning are similar in terms of accuracy in the diagnosis of MPM, although MRI is superior to CT scanning in depicting isolated foci of the chest wall and diaphragmatic invasion. MRI is superior to CT, both in the differentiation of malignant from benign pleural disease and in the assessment of chest wall and diaphragmatic involvement. Perfusion and diffusion MRI are innovative techniques used in the assessment of tumor cellularity and microvasculature and can be used utilized in quantitative and qualitative assessment of response to therapy. However, this difference has not been shown to confer any benefit in terms of overall staging. ${ }^{44-45}$ MPM typically shows high signal intensity on T1-weighted images and moderately high signal intensity on T2-weighted images. MRI and FDG-PET is useful in distinguishing between DPT and MPM (Figures 22,26).

\section{Ultrasonography}

Ultrasonography is useful in characterizing pleural effusions and evaluating pleural thickening or masses. Ultrasonography also facilitates image-guided pleural intervention (Figures 22,24). ${ }^{46}$ Ultrasonography ${ }^{47}$ has achieved diagnosis of round atelectasis.

\section{Nuclear Imaging}

Gallium- 67 has been used to quantitate lung parenchymal uptake to create an index of inflammatory activity in asbestosis.48 When combined with evidence of serum markers indicating inflammation-associated pulmonary collagen formation, the findings may provide a clinically useful algorithmic approach permitting an early diagnosis of asbestosis. MPM unrelated to asbestosis may also uptake Gallium-67. Gallium-67 study has been used to differentiate malignant from benign, asbestos-related pleural disease. Although experience is limited, the combina-

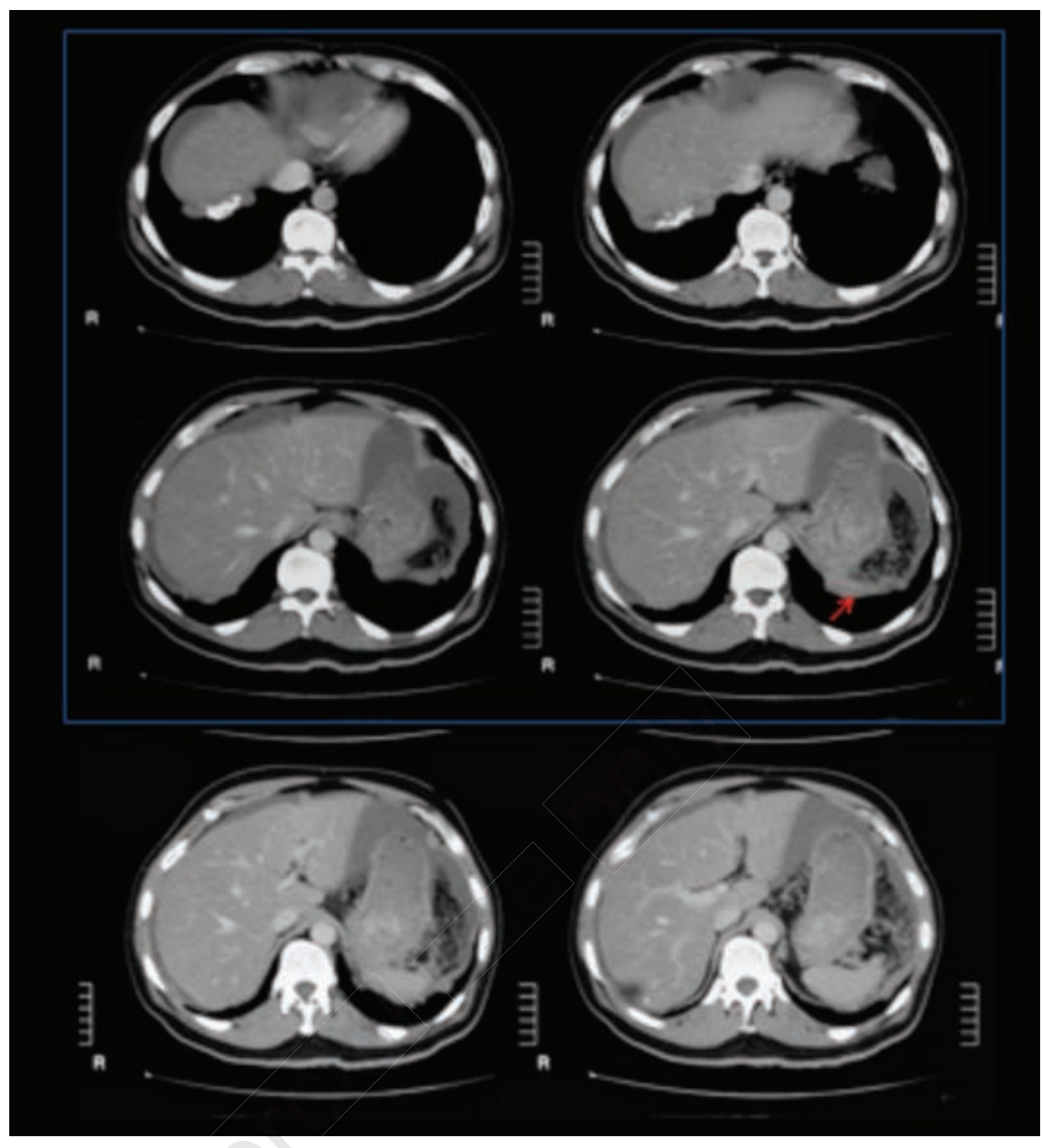

Figure 27. Peritoneal mesothelioma: This 55-year-old banker presented with vague abdominal discomfort. His CXR was clear. An abdominal ultrasound showed mild non-specific ascites. A contrast-enhanced CT scan of the abdomen shows peritoneal nodular thickening (arrow) associated with a mild ascites. Supra diaphragmatic and left paravertebral pleural plaques (arrows). On further interrogation revealed that, he had worked as a manager at a plumbing factory 30 years earlier.

tion of HRCT scanning, Gallium-67 scanning, and inflammatory serum marker testing may allow for an earlier diagnosis of asbestosis. ${ }^{48}$

FDG-PET is useful modality for the differentiation of benign from malignant lesions, for staging, and for monitoring response to therapy. PET-CT is superior to other imaging modalities in detecting more extensive disease involvement and identifying unsuspected occult distant metastases. ${ }^{41}$ FDG PET/CT carries an independent prognostic value. Survival in recurrent disease is dependent more on the intensity of uptake and on the pattern of metabolically active disease in FDG PET/CT (Figure 24).

\section{Conclusions}

ARLD is a serious potential risk in the developing countries where industrialization has just begun and it may take decades to remove the threat of asbestos. Recognition of the clin- ical, radiologic, and pathologic features of ARLD will be important for some years to come. Developing countries have a need to tighten health safety regulations in the work place. Research on non-invasive screening tests for ARLD needs to be pursued in earnest. Despite limitations, a CXR remains the initial imaging modality for the detection of ARLD. HRCT scanning is playing an increasingly important role in the diagnosis of diffuse interstitial lung disease. MRI is useful in depicting chest wall invasion with MPM. Both Ga-67 and FDG-PET can differentiate benign from malignant lesions. Ultrasonography has an important role in differentiating pleural thickening from pleural effusions and guiding pleural aspiration and biopsy.

\section{References}

1. Gardner MN, Brandt AM. "The doctors' 
choice is America's choice": the physician in US cigarette advertisements, 1930-1953. Am J Public Health 2006;96:222-32.

2. Bolton C, Richards A, Ebden P. Asbestosrelated disease. Hosp Med 2002;63:148-51.

3. Roggli VL, Vollmer RT. Twenty-five years of fiber analysis: what have we learned? Hum Pathol 2008;39:307-15.

4. Guidotti TL. Apportionment in asbestosrelated disease for purposes of compensation. Ind Health 2002;40:295-311.

5. Attanoos RL, Gibbs AR. Pseudomesotheliomatous carcinomas of the pleura: a 10-year analysis of cases from the Environmental Lung Disease Research Group, Cardiff. Histopathology 2003;43:44452.

6. Bard M, Debrosse D, Caliandro R, et al. [Current insights in malignant pleural mesothelioma]. Bull Cancer 2002;89:67-74.

7. Girling DJ, Muers MF, Qian W, Lobban D. Multicenter randomized controlled trial of the management of unresectable malignant mesothelioma proposed by the British Thoracic Society and the British Medical Research Council. Semin Oncol 2002;29:97101.

8. Puntoni R, Filiberti R, Cerrano PG, et al. Implementation of a molecular epidemiology approach to human pleural malignant mesothelioma. Mutat Res 2003;544:385-96.

9. Yilmaz UM, Utkaner G, Yalniz E, Kumcuoglu Z. Computed tomographic findings of environmental asbestos-related malignant pleural mesothelioma. Respirology 1998;3:33-8.

10. Jamrozik E, de Klerk N, Musk AW. Asbestosrelated disease. Intern Med J 2011;41:37280.

11. Gamsu G, Aberle DR, Lynch D. Computed tomography in the diagnosis of asbestosrelated thoracic disease. J Thorac Imaging 1989;4:61-7.

12. Tischoff I, Neid M, Neumann V, Tannapfel A. Pathohistological diagnosis and differential diagnosis. Recent Results Cancer Res 2011;189:57-78.

13. Tanrikulu AC, Abakay A, Kaplan MA, et al. A clinical, radiographic and laboratory evaluation of prognostic factors in 363 patients with malignant pleural mesothelioma. Respiration 2010;80:480-7.

14. Cagle PT, Allen TC. Pathology of the pleura: what the pulmonologists need to know. Respirology 2011;16:430-8.

15. Bean SM, Eloubeidi MA, Cerfolio R, et al. Endoscopic ultrasound-guided fine needle aspiration is useful for nodal staging in patients with pleural mesothelioma. Diagn Cytopathol 2008;36:32-7.

16. Kao SC, Reid G, van Zandwijk N, et al. Molecular biomarkers in malignant mesothelioma: state of the art. Pathology 2011;43:201-12.

17. Porcel JM. Pearls and myths in pleural fluid analysis. Respirology 2011;16:44-52.

18. Galbis JM, Mata M, Guijarro R, et al. Clinical-therapeutic management of thoracoscopy in pleural effusion: a groundbreaking technique in the twenty-first century. Clin Transl Oncol 2011;13:57-60.

19. Rahman NM, Gleeson FV. Image-guided pleural biopsy. Curr Opin Pulm Med 2008;14:331-6.

20. Peacock C, Copley SJ, Hansell DM. Asbestosrelated benign pleural disease. Clin Radiol 2000;55:422-32.

21. Chapman EA, Thomas PS, Yates DH. Breath analysis in asbestos-related disorders: a review of the literature and potential future applications. J Breath Res 2010;4:034001.

22. Perić I, Novak K, Barisić I, et al. Interobserver variations in diagnosing asbestosis according to the ILO classification. Arh Hig Rada Toksikol 2009;60:191-5.

23. McLoud TC. Conventional radiography in the diagnosis of asbestos-related disease. Radiol Clin North Am 1992;30:1177-89.

24. Whitman GJ, Niklason LT, Pandit M, et al. Dual-energy digital subtraction chest radiography: technical considerations. Curr Probl Diagn Radiol 2002;31:48-62.

25. Staples CA. Computed tomography in the evaluation of benign asbestos-related disorders. Radiol Clin North Am 1992;30: 1191207.

26. Woodring JH. Pleural effusion is a cause of round atelectasis of the lung. $\mathrm{J}$ Ky Med Assoc 2000;98:527-32.

27. Ak G, Metintas M, Metintas S, et al. Characteristics of lung cancer patients with asbestos-related radiological findings. Tuberk Toraks 2008;56:257-65.

28. Kao SC, Yan TD, Lee K, et al. Accuracy of diagnostic biopsy for the histological subtype of malignant pleural mesothelioma. J Thorac Oncol 2011;6:602-5.

29. Tiitola M, Kivisaari L, Huuskonen MS, et al. Computed tomography screening for lung cancer in asbestos-exposed workers. Lung Cancer 2002;35:17-22.

30. Tiitola M, Kivisaari L, Zitting A, et al. Computed tomography of asbestos-related pleural abnormalities. Int Arch 0ccup Environ Health 2002;75:224-8.

31. Epstein DM, Miller WT, Bresnitz EA, et al. Application of ILO classification to a population without industrial exposure: findings to be differentiated from pneumoconiosis. AJR Am J Roentgenol 1984;142:53-8.

32. Majurin ML, Varpula M, Kurki T, et al. Highresolution CT of the lung in asbestosexposed subjects. Comparison of low-dose and high-dose HRCT. Acta Radiol 1994;35:473-7.

33. Aberle DR. High-resolution computed tomography of asbestos-related diseases. Semin Roentgenol 1991;26:118-31.

34. Dujic Z, Tocilj J, Saric M. Early detection of interstitial lung disease in asbestos exposed non- smoking workers by mid-expiratory flow rate and high resolution computed tomography. Br J Ind Med 1991;48:663-4.

35. Huuskonen 0, Kivisaari L, Zitting K, et al. High-resolution computed tomography classification of lung fibrosis for patients with asbestos-related disease. Scand J Work Environ Health 2001;27:106-12.

36. Neri S, Boraschi P, Antonelli A, et al. Pulmonary function, smoking habits, and high resolution computed tomography (HRCT) early abnormalities of lung and pleural fibrosis in shipyard workers exposed to asbestos. Am J Ind Med 1996;30:588-95.

37. Murray KA, Gamsu G, Webb WR, et al. Highresolution computed tomography sampling for detection of asbestos-related lung disease. Acad Radiol 1995;2:111-5.

38. Gamsu G. Computed tomography and highresolution computed tomography of pneumoconioses. J Occup Med 1991;33:794-6.

39. Lynch DA, Gamsu G, Ray CS, Aberle DR. Asbestos-related focal lung masses: manifestations on conventional and high-resolution CT scans. Radiology 1988;169:603-7.

40. Roach HD, Davies GJ, Attanoos R, et al. Asbestos: when the dust settles an imaging review of asbestos-related disease. Radiographics 2002;22 Spec No:S167-84.

41. Gill RR, Gerbaudo VH, Sugarbaker DJ, Hatabu H. Current trends in radiologic management of malignant pleural mesothelioma. Semin Thorac Cardiovasc Surg 2009;21:111-20.

42. Chong S, Lee KS, Chung MJ, et al. Pneumoconiosis: comparison of imaging and pathologic findings. Radiographics 2006;26:59-77.

43. Jarad NA, Underwood SR, Rudd RM. Asbestos-related pericardial thickening detected by magnetic resonance imaging. Respir Med 1993;87:309-12.

44. Plathow C, Klopp M, Thieke C, et al. Therapy response in malignant pleural mesothelioma-role of MRI using RECIST, modified RECIST and volumetric approaches in comparison with CT. Eur Radiol 2008;18:163543.

45. Gill RR, Gerbaudo VH, Sugarbaker DJ, Hatabu H. Current trends in radiologic management of malignant pleural mesothelioma. Semin Thorac Cardiovasc Surg 2009;21:111-20.

46. Klein JS. Thoracic intervention. Curr Opin Radiol 1992;4:94-103.

47. Marchbank ND, Wilson AG, Joseph AE. Ultrasound features of folded lung. Clin Radiol 1996;51:433-7.

48. Cordasco EM, O'Donnell J, MacIntyre W, et al. Multiplane gallium tomography in assessment of occupational chest diseases. Am J Ind Med 1990;17:285-97. 